\title{
Towards Fatty Acid Determination in Human Milk by Attenuated Total Reflection Infrared Spectroscopy and Solvent-Free Lipid Separation
}

Christopher K. Akhgar ${ }^{1}$, Vanessa Nürnberger ${ }^{2}$, Marlene Nadvornik ${ }^{1}$, Victoria Ramos-Garcia ${ }^{3}$, Isabel Ten-Doménech ${ }^{3}$, Julia Kuligowski ${ }^{3}$, Andreas Schwaighofer ${ }^{1}$, Erwin Rosenberg ${ }^{1}$ and Bernhard Lendl ${ }^{1 *}$

${ }^{1}$ Institute of Chemical Technologies and Analytics, Technische Universität Wien, Getreidemarkt 9, 1060 Vienna, Austria

${ }^{2}$ Competence Center CHASE GmbH, Altenberger Straße 69, 4040 Linz, Austria

${ }^{3}$ Health Research Institute La Fe, Avenida Fernando Abril Martorell 106, 46026 Valencia, Spain.

*Corresponding author: Bernhard Lendl, Institute of Chemical Technologies and Analytics, Technische Universität Wien, Getreidemarkt 9, 1060 Vienna, Austria. Email: bernhard.lend1@tuwien.ac.at

(C) 2022 The Authors. Reuse is restricted to non-commercial and no derivative uses. This is the accepted version of the following article: C.K. Akhgar, V. Nürnberger, M. Nadvornik, V. Ramos-Garcia, I. Ten-Doménech, J. Kuligowski, A. Schwaighofer, E. Rosenberg, B. Lendl. “Towards Fatty Acid Determination in Human Milk by Attenuated Total Reflection Infrared Spectroscopy and Solvent-Free Lipid Separation", Applied Spectroscopy, which has been published in final form at https://doi.org/10.1177/00037028211065502. 


\begin{abstract}
This study introduces the first mid-infrared (IR) based method for determining the fatty acid composition of human milk. A representative milk lipid fraction was obtained by applying a rapid and solvent-free two-step centrifugation method. Attenuated total reflection-Fourier transform infrared (ATR-FTIR) spectroscopy was applied to record absorbance spectra of pure milk fat. The obtained spectra were compared to whole human milk transmission spectra, revealing the significantly higher degree of fatty acid related spectral features in ATR-FTIR spectra. Partial least squares (PLS) based multivariate regression equations were established by relating ATR-FTIR spectra to fatty acid reference concentrations, obtained with gas chromatography-mass spectrometry (GC-MS). Good predictions were achieved for the most important fatty acid sum parameters: saturated fatty acids $\left(\mathrm{SAT}, \mathrm{R}^{2} \mathrm{CV}=0.94\right)$, monounsaturated fatty acids (MONO, $\mathrm{R}^{2} \mathrm{CV}=0.85$ ), polyunsaturated fatty acids (PUFA, $\mathrm{R}^{2} \mathrm{CV}=0.87$ ), unsaturated fatty acids (UNSAT, $\mathrm{R}^{2} \mathrm{CV}=0.91$ ), short-chain fatty acids ( $\mathrm{SCFA}, \mathrm{R}^{2} \mathrm{CV}=0.79$ ), medium-chain fatty acids (MCFA, $\mathrm{R}^{2} \mathrm{CV}=0.97$ ) and long-chain fatty acids (LCFA, $\mathrm{R}^{2}{ }_{\mathrm{CV}}=0.88$ ). The PLS selectivity ratio (SR) was calculated in order to optimize and verify each individual calibration model. All mid-IR regions with high SR could be assigned to absorbances from fatty acids, indicating high validity of the obtained models.
\end{abstract}

Keywords: Mid-infrared spectroscopy, human milk, attenuated total reflection, partial least squares regression, fatty acids 


\section{Introduction}

Human milk is a complex mixture, containing a multitude of constituents that either can individually or in combination contribute to various health outcomes for the infant. ${ }^{1}$ Among the most important factors affecting human milk composition are the stage of lactation and term/preterm delivery. ${ }^{2}$ The typical macronutrient composition of term, mature human milk is 0.9$1.2 \mathrm{~g} / \mathrm{dL}$ for proteins, 3.2-3.6 g/dL for fat and 6.7-7.8 g/dL for lactose. ${ }^{3}$ Fatty acids represent the

most variable macronutrients, ${ }^{4}$ known to be additionally influenced by mothers diet, genetics, sociodemographic and environmental factors. ${ }^{5}$ Milk fat of European women typically contains approx. $35-40 \%$ saturated fatty acids (SAT), $45-50 \%$ monounsaturated fatty acids (MONO) and $15 \%$ polyunsaturated fatty acids (PUFA). ${ }^{6}$

Quantification of individual fatty acids from human milk is routinely performed by gas chromatography (GC), ${ }^{7}$ offering high accuracy and sensitivity. Some drawbacks are however, the associated high costs, significant time consumption and the essential derivatization step prior to analysis. Due to this work-intensive procedure, there is a need for more rapid, less expensive and high-throughput methods.

Mid-infrared (IR) spectroscopy provides detailed information about rotational-vibrational transitions of molecules, allowing identification and quantification of a wide range of biochemical components. ${ }^{8}$ Fourier-transform IR (FTIR) devices are the most established and widespread instrumentation available operating in this spectral region. For determination of the macronutrient composition of human milk, including total protein, total fat and lactose content, commercially available mid-IR based devices (e.g. MIRIS human milk analyzer, Uppsala, Sweden) that record absorbance spectra of whole milk in transmission mode are routinely employed at human milk banks. It has been shown that the hereby obtained results are acceptable for most measurement parameters. ${ }^{9}$ Moreover, Raman spectroscopy was applied to analyze the composition of human milk. ${ }^{10-12}$ As an alternative probing mode, attenuated total reflection (ATR) is commonly applied in FTIR spectroscopy. In this approach, the IR beam passes through an ATR element of high optical density, where it is totally reflected at the interface with an optically less dense sample. Through this reflection, an evanescent field is formed that can penetrate samples at typical depths between 0.5 and $2 \mu \mathrm{m} .{ }^{13}$ Previously, ATR-FTIR spectroscopy was applied to determine total protein, fat and carbohydrate content in human milk. ${ }^{14}$ The potential of FTIR spectroscopy for determining the fatty acid composition of bovine milk has been studied extensively. ${ }^{15}$ Here, reported methods 
include probing of whole milk, ${ }^{16}$ as well as measuring the extracted lipid fraction after solvent extraction. ${ }^{17}$ Method validation showed that solvent-free fat separation combined with ATR-FTIR spectroscopy also allows accurate bovine milk fatty acid profiling. ${ }^{18}$ Method validation showed that the thus obtained fraction possesses a representative fatty acid composition for milk from different species. ${ }^{19,20}$

However, to the best of our knowledge, no studies reporting mid-IR based determination of human milk fatty acids are available up to now. The aim of the present study is to show the potential of solvent-free lipid separation and ATR-FTIR spectroscopy for measuring the fatty acid composition of human milk. On this account, a rapid two-step centrifugation method was used to receive a pure lipid fraction without using organic solvents. ATR-FTIR absorbance spectra of the obtained lipid fraction were recorded and compared with spectra of whole human milk, recorded in FTIR transmission mode. Partial least squares (PLS) based multivariate calibration models were established in order to relate the ATR-FTIR spectra to fatty acid reference concentrations, measured with gas chromatography-mass spectrometry (GC-MS). The obtained individual PLS models were optimized by evaluating the importance of mid-IR wavenumbers for predicting the target variables. Based on eight human milk samples, the calculated statistical figures of merit clearly indicate high potential towards future high-throughput human milk fatty acid profiling.

\section{Material and Methods}

\section{Human Milk Samples}

Human milk samples $(N=8)$ were collected following the instructions of the hospital staff. Milk expression was accomplished with breast milk pumps following the standard operating procedure routinely employed at the hospital and the human milk bank. Prior to extraction, both removable parts of the breast milk pump and collection bottles were sterilized. In addition, mothers must wash their hands with soap and water and nipples with water. Extracted milk was stored at $-20^{\circ} \mathrm{C}$ until further processing.

\section{Human milk macronutrient analysis}

Human milk macronutrients including fat, carbohydrate, crude and true protein, total solids, and energy were determined using a MIRIS HMA ${ }^{\mathrm{TM}}$ (MIRIS AB, Uppsala Sweden). Determinations, 
quality control, and instrument calibration were performed following the standard operation procedure given by the manufacturer. ${ }^{21}$

\section{Lipid Separation}

Human milk lipids were separated by employing a previously reported centrifugation method. ${ }^{20}$ First, $30 \mathrm{~mL}$ sample aliquots were unfrozen at $4{ }^{\circ} \mathrm{C}$ overnight, followed by storing them at ambient temperature for a minimum of $20 \mathrm{~min}$. Second, they were centrifuged at $20{ }^{\circ} \mathrm{C}$ for $30 \mathrm{~min}$ at $17,800 \times \mathrm{g}$ in a Sigma 3-18k centrifuge (Sigma Laborzentrifugen GmbH, Osterode am Harz, Germany). The obtained upper phase, containing lipids, proteins and water was removed and transferred into microtubes, followed by a second centrifugation step at $20{ }^{\circ} \mathrm{C}$ for 20 min at $19,300 \times \mathrm{g}$. The thereby obtained pure fat layer of approximately $20-150 \mathrm{mg}$ was removed and analyzed with ATR-FTIR and GC-MS.

\section{GC-MS Analysis}

Sample solutions were prepared from the milk fat, isolated according to the previously described procedure, by dissolving $20 \mathrm{mg}$ per $\mathrm{ml}$ dichloromethane. Derivatization of fatty acids was carried out by transferring $50 \mu \mathrm{l}$ of this solution to a cooled vial ( $1.5 \mathrm{~mL}$ with $0.2 \mathrm{ml}$ low-volume insert). To the sample, $50 \mu \mathrm{l}$ each of the C17:0 internal standard solution and the $0.25 \mathrm{M}$ trimethylsulfonium hydroxide (TMSH) in methanol (Supelco, Vienna, Austria) derivatization reagent were added. Each sample was vortex mixed for $5 \mathrm{~s}$, and the derivatization reaction was brought to completion by heating to $70^{\circ} \mathrm{C}$ for $15 \mathrm{~min}$.

Quantitative GC-MS analysis and profiling of fatty acids was performed using a Shimadzu QP2010 Plus gas chromatograph with a single-quadrupole mass spectrometer (Shimadzu, Kyoto, Japan), equipped with a ZB-FAME column (30 m x $0.25 \mathrm{~mm}$ I.D. x $0.25 \mu \mathrm{m}$ film thickness) obtained from Phenomenex (Aschaffenburg, Germany). Split mode injection (split 100:1) of $1 \mu 1$ sample volumes was performed at $250{ }^{\circ} \mathrm{C}$ injector temperature using a Shimadzu AOC-5000 Plus autosampler. The septum purge flow was kept constant at $3 \mathrm{ml} / \mathrm{min}$ while the column flow was set at $2.14 \mathrm{ml} / \mathrm{min}$ initially under constant linear velocity mode. The oven program was: $40{ }^{\circ} \mathrm{C}(3.00$ $\mathrm{min}$ ), then increase of $10{ }^{\circ} \mathrm{C} / \mathrm{min}$ to $100{ }^{\circ} \mathrm{C}$ (no hold), and further at a rate of $2{ }^{\circ} \mathrm{C} / \mathrm{min}$ to $200{ }^{\circ} \mathrm{C}$ (no hold). Total run time was $59.00 \mathrm{~min}$. Both the transfer line and the ion source temperature were 
kept at a temperature of $200{ }^{\circ} \mathrm{C}$. Data acquisition was started only after a solvent delay of $2.7 \mathrm{~min}$, when the detector voltage was set to $1.05 \mathrm{kV}$. Data acquisition was performed in scan mode $(\mathrm{m} / z$ 35-350).

Quantitative analysis of FAME was performed by calibrating the system with a TraceCERT® Supelco 37-compound FAME mix (Supelco, Vienna, Austria). For this purpose, calibration levels between 20 and $600 \mathrm{mg} / \mathrm{L}$ were prepared. During data evaluation, the peak area of the quantifier ion is evaluated for each target compound, if the quantifier/qualifier ion peak ratio is within the pre-defined acceptance range. Detailed information on the retention times and the quantifier and qualifier ions used for each FAME have been reported previously. ${ }^{18}$

\section{FTIR Measurements}

A Bruker Tensor 37 FTIR spectrometer (Ettlingen, Germany) equipped with a mercury cadmium telluride (MCT) detector $\left(D^{*}=4 \times 10^{10} \mathrm{~cm} \mathrm{~Hz}^{0.5} \mathrm{~W}^{-1}\right.$ at $\left.9.2 \mu \mathrm{m}\right)$ was used for all mid-IR measurements. Data acquisition was performed by applying the following settings: spectral resolution of $2 \mathrm{~cm}^{-1}$, double-sided, forward-backward acquisition mode, scan range from 600 to $4000 \mathrm{~cm}^{-1}, 52 \mathrm{~s}$ measurement time (averaging 128 scans per spectrum). The final spectra were calculated by using a Blackman-Harris 3-term apodization function and a zero-filling factor of 2. By constantly flushing the spectrometer with dry air, the influence of atmospheric water vapor was reduced. ATR measurements were performed by transferring approximately $5 \mu \mathrm{L}$ of the extracted lipid fraction onto a Platinum ATR single-bounce element (Bruker, Ettlingen, Germany). After each measurement, the surface of the ATR element was cleaned with dichloromethane and isopropanol until the initial baseline signal was recovered. Reference measurements were performed by placing homogenized human milk into a $\mathrm{CaF}_{2}$ transmission cell with an optical path length of $50 \mu \mathrm{m}$ (as used in the commercially available MIRIS human milk analyzer). Data was acquired and analyzed with the spectroscopy software OPUS 7.2 (Bruker, Ettlingen, Germany).

\section{Multivariate Calibration Equations}

Multivariate calibration equations were calculated in MATLAB R2020a (Mathworks Inc., Nattick, MA, USA) using the PLS Toolbox 8.9 from Eigenvector Research Inc. (Wenatchee, WA, USA).

First, the recorded spectra of pure milk fat were treated with a preprocessing routine, combining 
mean centering and calculation of $2^{\text {nd }}$ derivative by applying a Savitzky Golay filter with a window of 15 points and polynomial order of 2. Partial least squares (PLS) regression models were created by relating the preprocessed ATR-FTIR spectra to the fatty acid concentrations measured with GCMS. The included spectral range was individually selected for each target variable, according to the PLS selectivity ratio (SR). ${ }^{22}$ The number of latent variables was determined by applying a leave-one-out cross-validation.

\section{Results and Discussion}

\section{Comparison between mid-IR Spectra of Whole Human Milk and Pure Lipid Fraction}

In the present study, human milk lipids were separated with a rapid two-step centrifugation method prior to ATR-FTIR analysis. ${ }^{20}$ Table I gives an overview of typical mid-IR absorbance bands of human milk fat. Figure 1 shows a comparison between a typical absorbance spectrum of pure lipid extract and a transmission spectrum of whole human milk, measured with a $\mathrm{CaF}_{2}$ cell and an transmission path of $50 \mu \mathrm{m}$. The whole milk spectrum includes absorbance bands from other major milk components such as proteins (amide II band: $1500-1600 \mathrm{~cm}^{-1}$ ) and sugars (approx. 1000-1480 $\left.\mathrm{cm}^{-1}\right)^{23}$ that overlap with significantly less intense fatty acid bands, thus adversely affecting the evaluation of those. For transmission measurements of whole milk, large optical path lengths ( $>30$ $\mu \mathrm{m})$ are necessary in order to avoid cell clogging. ${ }^{24}$ At these large optical path length, however, the spectral region between 1600 and $1700 \mathrm{~cm}^{-1}$ (e.g. $\mathrm{C}=\mathrm{C}$ stretch band at $1655 \mathrm{~cm}^{-1}$ ) is not accessible, due to total IR absorption through the $\mathrm{HOH}$ bending band of water. Furthermore, $\mathrm{CaF}_{2}$, the window material that is typically used for transmission measurements of milk, has a strong absorption starting at approximately $1000 \mathrm{~cm}^{-1},{ }^{25}$ meaning that absorbance bands at lower wavenumbers (e.g. CH out-of-plane band at $966 \mathrm{~cm}^{-1}$ and C-H rocking band at $722 \mathrm{~cm}^{-1}$ ) are inaccessible with this method. The described limitations of mid-IR transmission spectroscopy of whole milk clearly indicate the advantages of the approach based on ATR-FTIR spectroscopy and lipid separation in order to resolve more spectral details related to fatty acids. 
Table I. Overview of typical mid-IR absorbance bands of human milk fat ${ }^{26}$

\begin{tabular}{ccccc}
\hline Wavenumber/cm & Group & Functional Group & Mode of Vibration & Detectable in Whole Milk* \\
\hline 3005 & $\mathrm{C}-\mathrm{H}$ & $-\mathrm{C}=\mathrm{CH}-$ (cis) & symm. stretch & no \\
2953 & $\mathrm{C}-\mathrm{H}$ & $-\mathrm{CH}_{3}$ (aliphatic) & asymm. stretch & yes \\
2922 & $\mathrm{C}-\mathrm{H}$ & $-\mathrm{CH}_{2}$ - (aliphatic) & asymm. stretch & yes \\
2853 & $\mathrm{C}-\mathrm{H}$ & $-\mathrm{CH}_{2}$ - (aliphatic) & symm. stretch & yes \\
1743 & $\mathrm{C}=\mathrm{O}$ & $\mathrm{C}=\mathrm{O}$ ester & stretch & yes \\
1655 & $\mathrm{C}=\mathrm{C}$ & $\mathrm{C}=\mathrm{C}$ (unsaturated) & stretch & no \\
1462 & $\mathrm{C}-\mathrm{H}$ & $-\mathrm{CH}_{2}$ - (aliphatic) & scissoring & overlapping \\
1377 & $\mathrm{C}-\mathrm{H}$ & $-\mathrm{CH}_{3}$ (aliphatic) & symm. deformation & overlapping \\
1238 & $\mathrm{C}-\mathrm{H}$ & $-\mathrm{CH}_{2}-$ (aliphatic) & out-of-plane bend & overlapping \\
1162 & $\mathrm{C}-\mathrm{O}$ & $\mathrm{C}-\mathrm{O}$ ester & stretch & overlapping \\
966 & $\mathrm{C}-\mathrm{H}$ & $-\mathrm{C}=\mathrm{CH}-$ (trans) & out-of-plane bend & no \\
722 & $\mathrm{C}-\mathrm{H}$ & $-\mathrm{CH} \mathrm{H}_{2}$ - (aliphatic) & rocking & no \\
\hline
\end{tabular}

${ }^{\text {a }}$ Detectability of the absorbance band in a FTIR spectrum of whole human milk, recorded

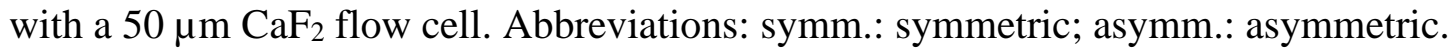

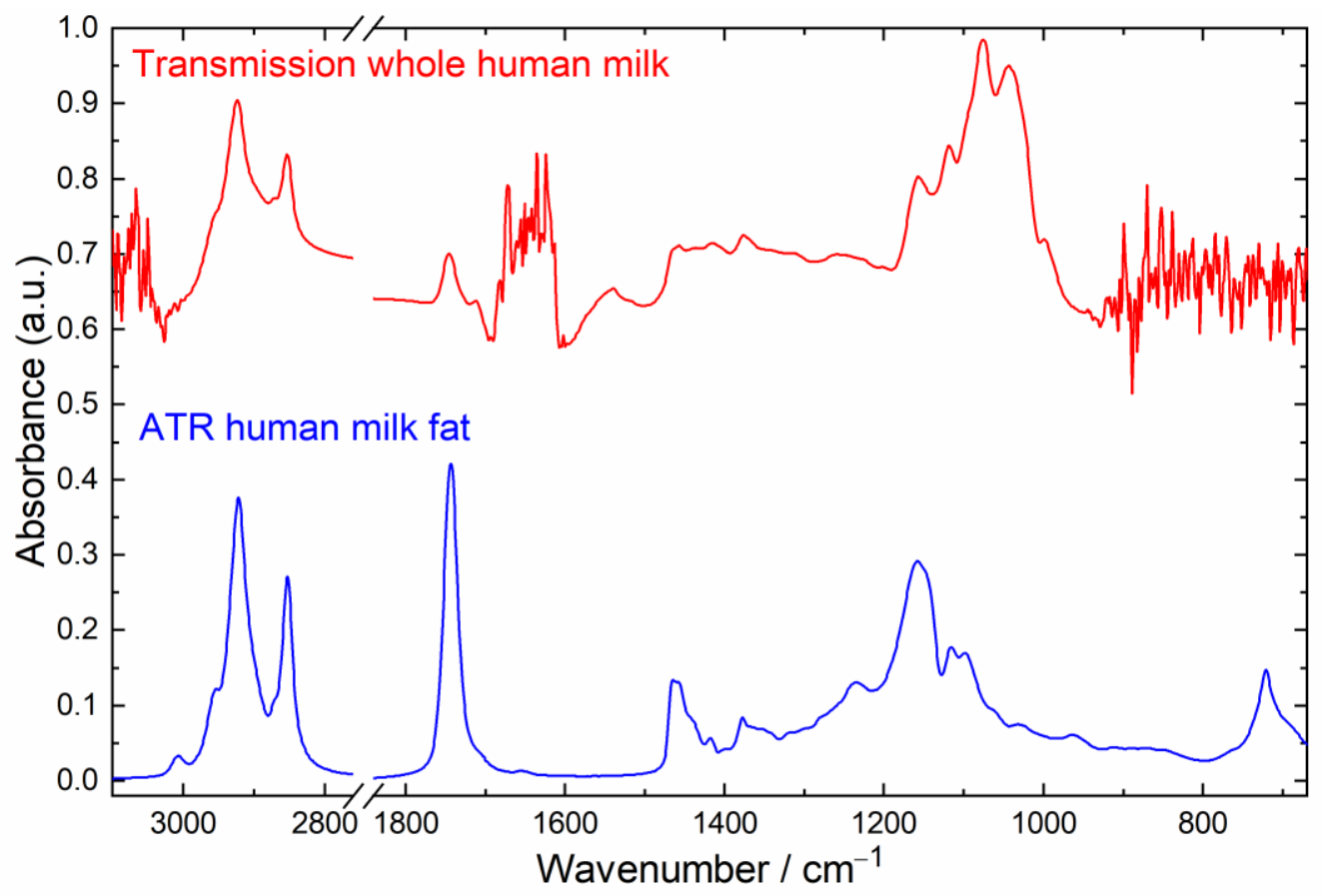

Figure 1. FTIR absorbance spectrum of whole human milk recorded with a CaF2 transmission cell (optical path length of $50 \mu \mathrm{m}$, red) and ATR spectrum of the separated lipid fraction (blue). The wavenumber region between $1850 \mathrm{~cm}-1$ and $2750 \mathrm{~cm}-1$ is not shown due to absence of significant information. 


\section{Fatty Acid Quantification}

A set of eight human milk samples from lactating mothers with mean (interquartile range, IQR) gestational and postnatal ages of 35+4 (7+4) weeks+days and 41 (131) days, respectively, was used in this study. Human milk samples were characterized with the MIRIS HMA providing median (IQR) concentrations of $2.8(1.1) \mathrm{g} / \mathrm{dL}$ for fat, $1.4(0.7)$ and $1.1(0.5) \mathrm{g} / \mathrm{dL}$ for crude and true protein, $7.7(0.6) \mathrm{g} / \mathrm{dL}$ for carbohydrate, 12.2 (1.9) g/dL total solid, and 63 (14) kcal/dL for energy.

In order to predict the most abundant fatty acid sum parameters, individual PLS models were calculated by relating the acquired ATR-FTIR spectra to GC-MS reference concentrations. Optimum results were achieved by first treating the spectra of pure milk fat with a preprocessing routine that combines calculation of the second derivative as well as mean centering. For each PLS model, the applied wavenumber range was individually selected (see next subchapter). The obtained statistical parameters for each prediction parameter are listed in Table II. The quality of calibration equations can be evaluated by the root mean square error of calibration (RMSEC) and calibration coefficient of determination $\left(\mathrm{R}^{2}\right)$.

Table II. Statistical figures of merit for each individual PLS model.

\begin{tabular}{lcccccc}
\hline & & \multicolumn{3}{c}{ g/100 g fat } & & \\
\cline { 4 - 6 } Fatty acid & LVs & Range & RMSEC & RMSECV & $\mathbf{R}^{\mathbf{2}}$ & $\mathbf{R}^{\mathbf{2}} \mathbf{C V}$ \\
\cline { 5 - 6 } SAT & 4 & $30.0-41.5$ & 0.19 & 1.1 & 0.998 & 0.937 \\
MONO & 3 & $35.6-54.7$ & 0.82 & 2.2 & 0.978 & 0.846 \\
PUFA & 3 & $12.7-28.5$ & 0.71 & 1.7 & 0.978 & 0.873 \\
UNSAT & 4 & $57.0-68.1$ & 0.22 & 1.3 & 0.997 & 0.906 \\
SCFA & 3 & $2.0-3.7$ & 0.10 & 0.26 & 0.968 & 0.786 \\
MCFA & 4 & $25.0-34.7$ & 0.17 & 0.57 & 0.997 & 0.969 \\
LCFA & 3 & $59.2-71.4$ & 0.71 & 1.5 & 0.972 & 0.877 \\
\hline
\end{tabular}

Abbreviations: LVs: latent variables; RMSEC: root mean square error of calibration; RMSECV: root mean square error of cross-validation; $\mathrm{R}^{2}$ : calibration coefficient of determination; $\mathrm{R}^{2} \mathrm{CV}$ : cross-validation coefficient of determination. SAT: saturated fatty acids; MONO: monounsaturated fatty acids; PUFA: polyunsaturated fatty acids; UNSAT: unsaturated fatty acids; SCFA: short-chain fatty acids (C4-C10); MCFA: medium-chain fatty acids (C12-C16); LCFA: long-chain fatty acids (C17 and higher). 
In order to assess the prediction efficiency, leave-one-out cross-validation was applied, providing the root mean square error of cross-validation (RMSECV) and the cross-validation coefficient of determination $\left(\mathrm{R}^{2} \mathrm{CV}\right)$. The number of latent variables (LVs) was selected by plotting the RMSEC and RMSECV versus the number of applied latent variables (see Figure S1). Excellent prediction efficiencies were obtained for SAT $\left(\mathrm{R}^{2} \mathrm{CV}=0.94\right)$ and unsaturated fatty acids (UNSAT, $\mathrm{R}^{2} \mathrm{CV}=0.91$ ). Further sub-classification showed good predictions for MONO $\left(\mathrm{R}^{2} \mathrm{CV}=0.85\right)$ and PUFA $\left(\mathrm{R}^{2} \mathrm{CV}=0.87\right)$. Prediction results concerning concentrations of fatty acids with different chain-length showed excellent results for medium-chain fatty acids (MCFA, C12-C16, $\mathrm{R}^{2} \mathrm{CV}=0.97$ ) as well as good results for short-chain fatty acids (SCFA, $\mathrm{C} 4-\mathrm{C} 10, \mathrm{R}^{2} \mathrm{CV}=0.79$ ) and long-chain fatty acids (LCFA, $\mathrm{C} 17$ and higher, $\mathrm{R}^{2} \mathrm{CV}=0.88$ ). Figure 2 highlights the quality of the achieved models by displaying the relationship between GC-MS reference concentrations and prediction results from the cross-validation on using SAT and MCFA as examples. The obtained concentrations for the fatty acid sum parameters also agree well with the typical human milk lipid composition of European women. ${ }^{4}$

In the present study, only a limited number of eight human milk samples was available. However, it was shown before that a higher number of samples can clearly improve the prediction efficiencies of fatty acids in bovine milk by IR spectroscopy combined with PLS quantification. ${ }^{16}$ Thus, even better results and also predictions for individual fatty acids can be expected when more
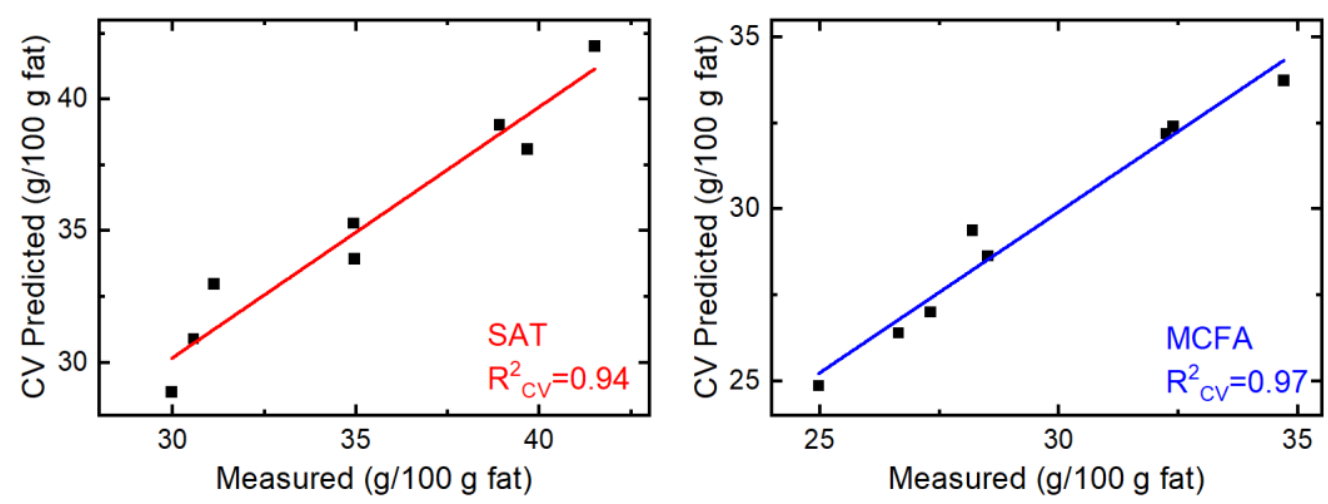

Figure 2. Measured (from GC-MS) versus predicted (from ATR-FTIR spectra and PLS crossvalidation) relative fatty acid content on the example of saturated fatty acids (SAT, left) and medium-chain fatty acids (MCFA, right). 
samples are available. A further important factor that might affect the performance of mid-IR based determination of the milk fatty acid composition are possible covariation structures between individual fatty acid concentrations and total fat content. It has been reported that the good prediction efficiencies for fatty acids in whole bovine milk are probably based on these covariation structures when absolute fatty acid concentrations are investigated. ${ }^{27}$ The applied approach inherently eliminates such covariation structures by measuring a representative fraction of pure milk lipids instead of whole milk samples. ${ }^{18,27}$

\section{Selection of Spectral Regions According to the Selectivity Ratio}

In order to optimize each individual PLS model, the selectivity ratio (SR) was calculated. A detailed explanation of the SR can be found in literature. ${ }^{22,28}$ In brief, it can be described as the ratio between explained and unexplained variance for each variable in the dataset, meaning that important variables for predicting target parameters can be identified. Previously, the SR has been demonstrated to be a strong tool for visualizing mid-IR regions with high correlation to PLS prediction parameters. ${ }^{18,29}$

In the present study, the included wavenumber range was individually optimized for each PLS model. Figure 3 depicts the included mid-IR regions: wavenumbers with low SR are displayed in bright grey whereas wavenumbers with high SR are highlighted in black. Moreover, Figures S2S8 show loading plots for the individual LVs of each PLS model. The SR and the loadings both indicate that all wavenumbers with high relevance can be related to fatty acid absorption bands listed in Table I. Relating important wavenumbers to specific absorbance bands is the main way to verify that the calculated PLS models are based on absorbance signals from fatty acids instead of coincidental correlations. It should be noted that a large part of these important wavenumbers is either completely inaccessible or highly overlapping with absorbances arising from other human milk constituents in transmission spectra of whole milk. Thus, the advantage of the applied approach based on lipid separation and ATR-FTIR spectroscopy is clearly visible.

[Insert Figure 3 here] 


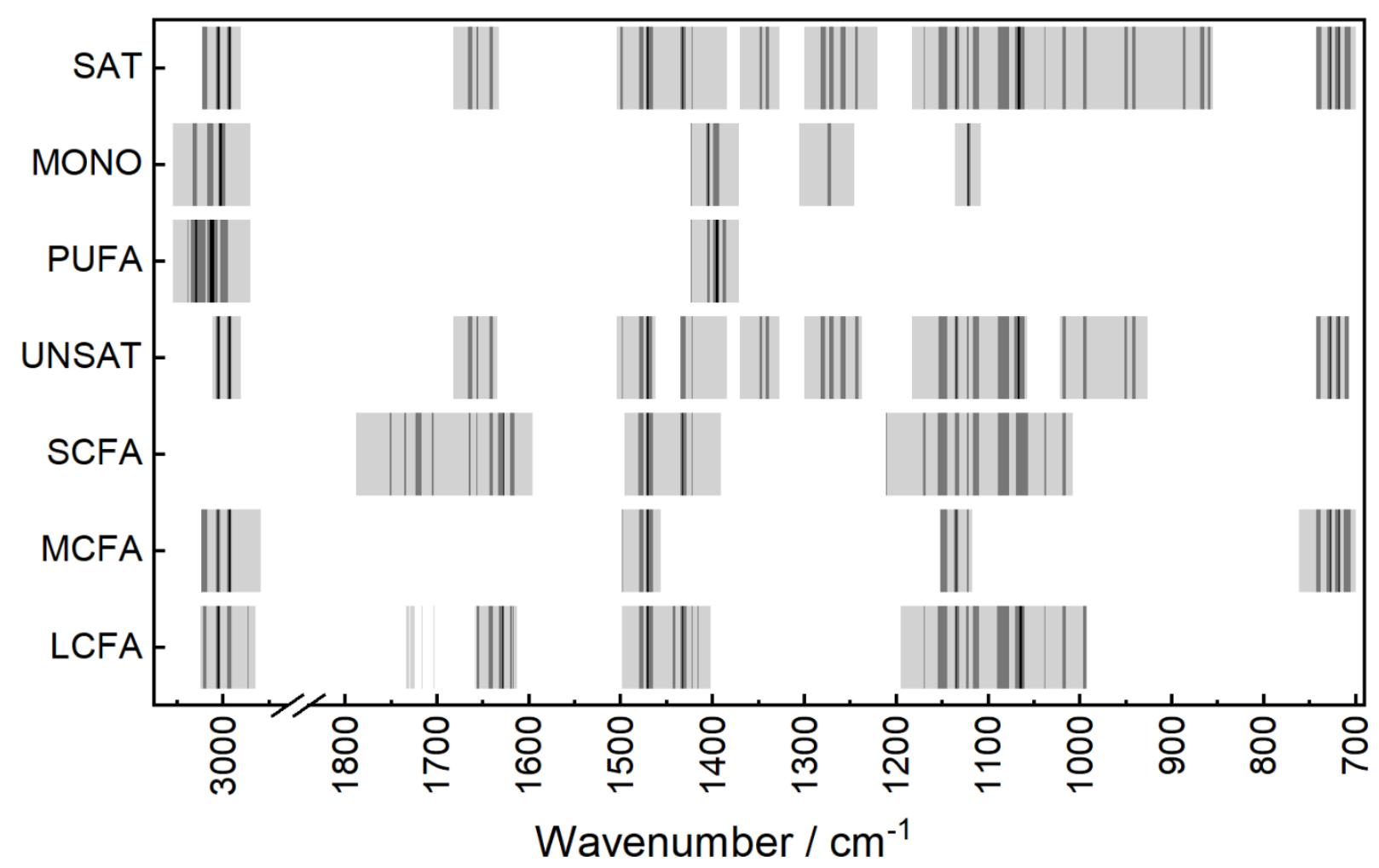

Figure 3. Included wavenumber regions for each individual calibration equation, according to the selectivity ratio (SR). SR of 0 to 0.5 : bright grey; 0.5 to 5 : dark grey; 5 to 15 : black. The spectral range between 1850 and $2950 \mathrm{~cm}^{-1}$ is not shown due to absence of significant information.

\section{Conclusion}

In this work, the first approach based on mid-IR spectroscopy for determining the human milk fatty acid composition was presented. A representative lipid fraction was obtained by a solventfree two-step centrifugation method, which was consequently measured with ATR-FTIR spectroscopy. The advantage of the fat separation step was demonstrated in the higher number of accessible spectral features from fatty acids, compared to transmission spectra of whole milk. PLS models, calculated by relating mid-IR spectra to GC-MS reference values showed good prediction accuracies for the important group parameters SAT, MONO, PUFA, SCFA, MCFA and LCFA. The SR was calculated to assign important wavenumber regions to spectral features arising from fatty acid absorbance. Based on a dataset of eight samples, the achieved statistical figures of merit show 
the high potential of the applied approach for future high-throughput human milk fatty acid profiling. We anticipate that even better results can be obtained, when a higher number of samples is available. Then prediction of individual fatty acids can also be expected, as previously shown for bovine milk. ${ }^{18}$

\section{Declaration of Conflicting Interests}

The authors declare no conflict of interest.

\section{Funding}

The authors are grateful to lactating mothers who agreed to participate in this study and to María Gormaz, Amparo Ramón, and Antonia Gálvez from the human milk bank at the University and Polytechnic Hospital La Fe for their support. The authors are also grateful to Prof. Oliver Spadiut and Julian Ebner from the Institute of Chemical, Environmental and Bioscience Engineering (Technische Universität Wien) for providing access to the applied centrifuge. This work was funded by the European Union's Horizon 2020 Research and Innovation Programme through the Nutrishield project (https://nutrishield-project.eu/) [Grant Agreement No 818110] as well as the COMET Centre CHASE, funded within the COMET - Competence Centers for Excellent Technologies programme by the BMK, the BMDW and the Federal Provinces of Upper Austria and Vienna. The COMET programme is managed by the Austrian Research Promotion Agency (FFG). ITD and JK received funding from the Instituto de Salud Carlos III, Ministry of Economy and Competitiveness, Spain [grant numbers CD19/00176 and CP16/00034].

\section{Ethical Approval}

The study was approved by the Ethics Committee for Biomedical Research of the Health Research

Institute La Fe, University and Polytechnic Hospital La Fe (Valencia, Spain) with registry \#2019289-1 and all methods were performed in accordance with relevant guidelines and regulations. Written informed consent was obtained from registered, healthy donors admitted after routine screening and interview to the human milk bank at the University and Polytechnic Hospital La Fe. 


\section{References}

1. D. Munblit, D.G. Peroni, A. Boix-Amorós, P.S. Hsu, B. Van't Land, M.C.L. Gay, A. Kolotilina, C. Skevaki, R.J. Boyle, M.C. Collado, J. Garssen, D.T. Geddes, R. Nanan, C. Slupsky, G. Wegienka, A.L. Kozyrskyj, J.O. Warner. "Human Milk and Allergic Diseases: An Unsolved Puzzle". Nutrients 2017. 9(8): 894.

2. M.-Y. Chung. "Factors Affecting Human Milk Composition". Pediatr. Neonatol. 2014. 55(6): 421-422.

3. O. Ballard, A.L. Morrow. "Human Milk Composition: Nutrients and Bioactive Factors". Pediatr. Clin. N. Am. 2013. 60(1): 49-74.

4. B. Koletzko, M. Rodriguez-Palmero, H. Demmelmair, N. Fidler, R. Jensen, T. Sauerwald. "Physiological Aspects of Human Milk Lipids". Early Hum. Dev. 2001. 65(Supp. 2): 3-18.

5. K. Miliku, Q.L. Duan, T.J. Moraes, A.B. Becker, P.J. Mandhane, S.E. Turvey, D.L. Lefebvre, M.R. Sears, P. Subbarao, C.J. Field, M.B. Azad. "Human Milk Fatty Acid Composition Is Associated with Dietary, Genetic, Sociodemographic, and Environmental Factors in the Child Cohort Study". Am. J. Clin. Nutr. 2019. 110(6): 1370-1383.

6. B. Koletzko. "Human Milk Lipids". Ann. Nutr. Metab. 2016. 69(suppl 2): 27-40.

7. C. Cruz-Hernandez, S. Goeuriot, F. Giuffrida, S.K. Thakkar, F. Destaillats. "Direct Quantification of Fatty Acids in Human Milk by Gas Chromatography". J. Chromatogr. A 2013. 1284: 174-179.

8. A. Schwaighofer, M. Brandstetter, B. Lendl. "Quantum Cascade Lasers (Qcls) in Biomedical Spectroscopy". Chem. Soc. Rev. 2017. 46(19): 5903-5924.

9. G. Fusch, N. Rochow, A. Choi, S. Fusch, S. Poeschl, A.O. Ubah, S.-Y. Lee, P. Raja, C. Fusch. "Rapid Measurement of Macronutrients in Breast Milk: How Reliable Are Infrared Milk Analyzers?". 2015. 34(3): 465-476.

10. E. Motta, R. Zângaro, L. Silveira. "Quantitative Determination of the Human Breast Milk Macronutrients by near-Infrared Raman Spectroscopy". Proc. SPIE 2012. 8229.

11. R. Ullah, S. Khan, S. Javaid, H. Ali, M. Bilal, M. Saleem. "Raman Spectroscopy Combined with a Support Vector Machine for Differentiating between Feeding Male and Female Infants Mother's Milk". Biomed. Opt. Express 2018. 9(2): 844-851.

12. N. Argov, S. Wachsmann-Hogiu, S.L. Freeman, T. Huser, C.B. Lebrilla, J.B. German. "SizeDependent Lipid Content in Human Milk Fat Globules". J. Agric. Food Chem. 2008. 56(16): 7446-7450.

13. G. Ramer, B. Lendl. "Attenuated Total Reflection Fourier Transform Infrared Spectroscopy". In: R. Meyers and R. Meyers, editors. Encyclopedia of Analytical Chemistry. Hoboken, USA: John Wiley \& Sons, 2013.

14. K. Bērziņš, S.D.L. Harrison, C. Leong, S.J. Fraser-Miller, M.J. Harper, A. Diana, R.S. Gibson, L.A. Houghton, K.C. Gordon. "Qualitative and Quantitative Vibrational Spectroscopic Analysis of Macronutrients in Breast Milk". Spectrochim. Acta A 2021. 246: 118982.

15. M. De Marchi, V. Toffanin, M. Cassandro, M. Penasa. "Invited Review: Mid-Infrared Spectroscopy as Phenotyping Tool for Milk Traits". J. Dairy Sci. 2014. 97(3): 1171-1186. 
16. M.J.M. Rutten, H. Bovenhuis, K. Hettinga, H.J.F. Valenberg, J. Arendonk. "Predicting Bovine Milk Fat Composition Using Infrared Spectroscopy Based on Milk Samples Collected in Winter and Summer". J. Dairy Sci. 2009. 92(12): 6202-6209.

17. I. Stefanov, V. Baeten, O. Abbas, B. Vlaeminck, B. De Baets, V. Fievez. "Evaluation of FtNir and Atr-Ftir Spectroscopy Techniques for Determination of Minor Odd- and BranchedChain Saturated and Trans Unsaturated Milk Fatty Acids". J. Agric. Food Chem. 2013. 61(14): 3403-3413.

18. C.K. Akhgar, V. Nürnberger, M. Nadvornik, M. Velik, A. Schwaighofer, E. Rosenberg, B. Lendl. "Fatty Acid Prediction in Bovine Milk by Attenuated Total Reflection Infrared Spectroscopy after Solvent-Free Lipid Separation". MDPI Foods 2021. 10(5): 1054.

19. S. Feng, A.L. Lock, P.C. Garnsworthy. "Technical Note: A Rapid Lipid Separation Method for Determining Fatty Acid Composition of Milk". J. Dairy Sci. 2004. 87(11): 3785-3788.

20. P. Luna, M. Juárez, M.A. Fuente. "Validation of a Rapid Milk Fat Separation Method to Determine the Fatty Acid Profile by Gas Chromatography". J. Dairy Sci. 2005. 88(10): 33773381 .

21. Miris. "HMA User Manual". 2020. https://www.mirissolutions.com/media/839b5db2-335e4170-9284-f2d00a6295d8 May 262021.

22. M. Farrés, S. Platikanov, S. Tsakovski, R. Tauler. "Comparison of the Variable Importance in Projection (Vip) and of the Selectivity Ratio (Sr) Methods for Variable Selection and Interpretation". J. Chemom. 2015. 29(10): 528-536.

23. A. Kohler, N. Afseth, K. Jørgensen, Å. Randby, H. Martens. "Quality Analysis of Milk by Vibrational Spectroscopy". In: C. Jim and G. Peter, editors. Handbook of Vibrational Spectroscopy. Hoboken, USA: John Wiley \& Sons, 2010.

24. A. Schwaighofer, B. Lendl. "Quantum Cascade Laser-Based Infrared Transmission Spectroscopy of Proteins in Solution". In: Y. Ozaki, M. Baranska, I.K. Lednev and B.R. Wood, editors. Vibrational Spectroscopy in Protein Research. Academic Press, 2020.

25. T.G. Mayerhöfer, S. Pahlow, U. Hübner, J. Popp. "CaF2: An Ideal Substrate Material for Infrared Spectroscopy?". Anal. Chem. 2020. 92(13): 9024-9031.

26. M. Safar, D. Bertrand, P. Robert, M.-F. Devaux, C. Genot. "Characterization of Edible Oils, Butters and Margarines by Fourier Transform Infrared Spectroscopy with Attenuated Total Reflectance". J. Am. Oil Chem. Soc. 1994. 71(4): 371-377.

27. C. Eskildsen, M. Rasmussen, S. Engelsen, L. Larsen, N.A. Poulsen, T. Skov. "Quantification of Individual Fatty Acids in Bovine Milk by Infrared Spectroscopy and Chemometrics: Understanding Predictions of Highly Collinear Reference Variables". J. Dairy Sci. 2014. 97(12): 7940-7951.

28. O.M. Kvalheim. "Variable Importance: Comparison of Selectivity Ratio and Significance Multivariate Correlation for Interpretation of Latent-Variable Regression Models". J. Chemom. 2020. 34(4): e3211.

29. A.C. V. D. dos Santos, R. Heydenreich, C. Derntl, A.R. Mach-Aigner, R.L. Mach, G. Ramer, B. Lendl. "Nanoscale Infrared Spectroscopy and Chemometrics Enable Detection of Intracellular Protein Distribution". Anal. Chem. 2020. 92(24): 15719-15725. 